\title{
Siderophore-Mediated Iron Transport: Crystal Structure of FhuA with Bound Lipopolysaccharide
}

\author{
Andrew D. Ferguson, Eckhard Hofmann, James W. Coulton, \\ Kay Diederichs, Wolfram Welte*
}

\begin{abstract}
FhuA, the receptor for ferrichrome-iron in Escherichia coli, is a member of a family of integral outer membrane proteins, which, together with the energytransducing protein TonB, mediate the active transport of ferric siderophores across the outer membrane of Gram-negative bacteria. The three-dimensional structure of FhuA is presented here in two conformations: with and without ferrichrome-iron at resolutions of 2.7 and 2.5 angstroms, respectively. FhuA is a $\beta$ barrel composed of 22 antiparallel $\beta$ strands. In contrast to the typical trimeric arrangement found in porins, FhuA is monomeric. Located within the $\beta$ barrel is a structurally distinct domain, the "cork," which mainly consists of a four-stranded $\beta$ sheet and four short $\alpha$ helices. A single lipopolysaccharide molecule is noncovalently associated with the membrane-embedded region of the protein. Upon binding of ferrichrome-iron, conformational changes are transduced to the periplasmic pocket of FhuA, signaling the ligand-loaded status of the receptor. Sequence homologies and mutagenesis data are used to propose a structural mechanism for TonB-dependent siderophore-mediated transport across the outer membrane.
\end{abstract}

Iron is universally required by all living cells. However, in aerobic environments, iron is found as highly insoluble ferric hydroxide complexes, which are forms that severely limit the bioavailability of iron (1). To acquire iron, microorganisms synthesize and secrete siderophores, compounds that chelate ferric iron and thereby form soluble iron complexes. In Gram-negative bacteria, all essential ions and nutrients are transported across the cell envelope in discrete steps. Transport across the cytoplasmic membrane is an energy-dependent high-affinity process, whereas transport across the outer membrane is primarily mediated by passive diffusion through nonspecific or substrate-specific porins (2). Because siderophore-iron complexes are found at exceedingly low concentrations in the external media, their rate of passive diffusion across the outer membrane is insufficient for supporting the requirements of cellular growth. Therefore, a class of high-affin-

A. D. Ferguson is in the Department of Microbiology and Immunology, McGill University, 3775 University Street, Montreal, Quebec, Canada H3A 2B4, and Fakultät für Biologie, Universität Konstanz, M656, Konstanz, Germany D-78457. E. Hofmann, K. Diederichs, and W. Welte are in the Fakultät für Biologie, Universität Konstanz, M656, Konstanz, Germany D-78457. J. W. Coulton is in the Department of Microbiology and Immunology, McGill University, 3775 University Street, Montreal, Quebec, Canada H3A 2B4

*To whom correspondence should be addressed. Email: wolfram.welte@uni-konstanz.de ity siderophore receptors exists within the outer membrane. They bind specific siderophore-iron complexes and promote their active transport into the periplasm, exploiting the electrochemical potential of the cytoplasmic membrane that is transduced to the outer membrane by the TonB-ExbB-ExbD complex. All TonB-dependent receptors possess a short sequence of residues at the $\mathrm{NH}_{2}$-terminus, which is termed the TonB box $(1,3)$. It has been proposed that this region functions as a mediator of the physical interaction between TonB and TonB-dependent receptors. TonB spans the periplasmic space and physically interacts with siderophore receptors, resulting in energy transduction by a mechanism that is common to all TonB-dependent receptors (3).

FhuA in the outer membrane of Escherichia coli (4) is the receptor for ferrichrome-iron. In addition to binding ferrichrome-iron, FhuA also functions as the primary receptor for the structurally related antibiotic albomycin, for several bacteriophages (T1, T5, UC-1, and $\phi 80$ ), for the peptide antibiotic microcin 25, and for the bacterial toxin colicin M. Because the deletion of a surface-located linear sequence converted FhuA from an energy-dependent receptor into a general diffusion channel, it was concluded that FhuA (5) and other TonB-dependent receptors (6) act as ligand-specific gated porins. When wild-type FhuA was incorporated into an artificial lipid bilayer, it did not form channels. However, the addition of bacteriophage T5 re- sulted in the formation of stable, high-conductance ion channels, which were electrically similar but not identical to those observed with the channel-forming mutants of FhuA (7).

General description. The $\mathrm{x}$-ray structure (Table 1) of FhuA is composed of a $\mathrm{COOH}-$ terminal $\beta$-barrel domain (residues 161 to 723 ) and an $\mathrm{NH}_{2}$-terminal cork domain (residues 1 to 160), which fills the barrel interior (Fig. 1, A and $\mathrm{B})$. According to a search through a database of protein structures $(8)$, the fold of the cork domain has not been observed. In contrast to the typical trimeric arrangement found in porins, FhuA is monomeric. The barrel is formed by 22 antiparallel transmembrane $\beta$ strands ( $\beta 1$ through $\beta 22$ ). Loops connect adjacent strands; there are short periplasmic turns (T1 through T10) and longer surface-located loops (L1 through L11) (Fig. 1A). The FhuA barrel is larger than any barrel formed by the porins-it is $69 \AA$ in height and has an elliptical cross section of 46 by $39 \AA$ (Fig. 1, A and B). In common with other membrane proteins, two girdles of aromatic residues mark the boundary of an apolar cylindrical zone on the barrel surface (Fig. 1A). They are positioned to extend into the lipid bilayer and delineate the border between the lipid hydrocarbon chains and the polar head groups. The distance ( $34 \AA$ ) from the upper aromatic girdle to the apex of L4 and the distance to the apices of other surfacelocated loops of FhuA are substantially larger in comparison with the equivalent distance in the known crystallographic structures of porins (Fig. 1A). This feature may facilitate the use of these loops for the attachment of FhuA-specific bacteriophages. Porins, which have much shorter surface-located loops, also function as receptors for bacteriophages (2).

The single lipopolysaccharide (LPS) molecule that is noncovalently associated with the membrane-embedded outer surface of FhuA exhibits the expected chemical structure for E. coli K-12 LPS (9). Specifically, lipid A is composed of two linked phosphorylated glucosamines and six fatty acid chains, the inner core possesses two octose and two heptose residues, and the outer core contains three hexose residues. The LPS molecule is positioned so that the glucosamine moieties are placed slightly above the upper aromatic girdle. Five of the six alkyl chains are closely apposed with the barrel surface and are parallel to the barrel axis, as expected for the chains of the external LPS monolayer (Fig. 1A) (10).

The cork domain, consisting mainly of a mixed four-stranded $\beta$ sheet ( $\beta$ A through $\beta D)$, extends from the periplasm to the ferrichrome-iron binding site (Fig. 1A). The electron density permits tracing of the cork domain beginning at $\mathrm{Glu}^{19}$. The cork domain is arranged in the barrel with the $\beta$ sheet plane inclined by $\sim 45^{\circ}$ to the membrane normal, so that it sterically occludes most of the cross 
section of the barrel (Fig. 1, A and B). The presence of the cork domain suggests that the direct passage of ferrichrome-iron and small molecules through FhuA is not possible. This agrees with the finding that FhuA that has been reconstituted into planar lipid bilayers shows no channel conductance $(5,7)$. The cork domain is connected to the barrel wall by extensive hydrogen bonding. The number of hydrogen bonds observed in the FhuA-ferrichrome-iron complex is slightly reduced in comparison with FhuA in the absence of ligand. Given the large buried surface area $\left(5000 \AA^{2}\right)$ between the inner barrel wall and the cork domain, we consider it unlikely that the entire cork domain detaches for ferrichrome-iron transport, for channel formation, or as a result of an interaction with TonB.

The cork domain delineates a pair of pockets within FhuA. The larger external pocket is open to the external environment and is restricted by barrel strands, surfacelocated loops, and cork domain apices A, B, and $\mathrm{C}$ (Fig. 1, A and B). The boundaries of the smaller periplasmic pocket are the barrel, cork domain loops, and the four-stranded $\beta$ sheet (Fig. 1A).

The ferrichrome-iron binding site and the external pocket. Located in the external pocket of the FhuA-ferrichrome-iron complex is a single ferrichrome-iron molecule. The binding site for ferrichrome-iron is situated slightly above the external outer membrane interface
(Fig. 1, A and B). Residues from apices A, B, and $\mathrm{C}$ of the cork domain and the barrel domain make direct hydrogen bonds or are in van der Waals contact with ferrichrome-iron atoms (Fig. 2). These residues are strongly conserved as assigned by the sequence alignment of ferrichrome-iron receptors from $E$. coli, Pantoea agglomerans, Salmonella paratyphi strain B, and Salmonella typhimurium $(11,12)$. Moreover, two water molecules have been identified in the binding site for ferrichrome-iron and may mediate the formation of additional hydrogen bonds between the ligand and FhuA (Fig. 3). Thus, the ferrichrome-iron binding site is coated with a tailored and complementary pattern of residues that tightly bind the ligand (dissociation constant $\left.K_{\mathrm{D}}, 0.2 \mu \mathrm{M}\right)(1,13)$. The deletion of residues 236 to 248 from L3 resulted in the loss of ferrichrome-iron uptake (12), a result that is in accord with the composition of the binding site. Although none of the residues within L4 (residues 318 to 339) contribute directly to the high-affinity binding of ferrichrome-iron, the conformation of this loop is critical for the targeting of the ligand to its binding site (Fig. 1, A and B) (14). The inner walls of the external pocket, surface-located loops, and barrel strands from the ferrichromeiron binding site to the external opening are lined by numerous aromatic residues (15). Ferrichrome-iron interacts favorably with aromatic residues, because it can be extracted from fungal extracts with benzoyl alcohol (16). Hydrox- amate-type siderophores such as ferrichrome are uncharged at physiological $\mathrm{pH}$ and are not inherently hydrophobic. We propose that the interaction of aromatic residues with ferrichrome-iron involves electrostatic interactions between the quadropole moment of the $\pi$ electron system (17) and the dipoles of surfacelocated peptide bonds. Accordingly, the aromatic residues lining the inner walls of the external pocket function to extract ferrichromeiron from the external medium; those found in the ferrichrome-iron binding site contribute to the high-affinity binding of ferrichrome-iron.

Ferrichrome-iron-induced conformational changes and transmembrane signaling. Comparing the structure of FhuA to its complex with ferrichrome-iron reveals two distinct conformations: the ligand-free and ligand-loaded conformations. In the barrel domain, the coordinates of the backbone atoms of FhuA and its complex with ferrichrome-iron are very similar (root mean square deviation, $0.42 \AA$ ), except for minor differences in the periplasmic turns $\mathrm{T} 8$ and T9 (Fig. 4). Key differences between the structures are localized in the cork domain. In the ferrichrome-iron binding site, an induced fit mechanism is observed. Apex B (residues 98 to 100 ) is translated $1.7 \AA$ upward toward ferrichrome-iron, resulting in the formation of multiple hydrogen bonds with the ligand. All loops of the cork domain between apex A and the periplasmic pocket follow this trans-

Fig. 1. The FhuA-ferrichrome-iron complex and a single LPS molecule noncovalently associated with its membrane-embedded surface. (A) FhuA in ribbon representation. The barrel is colored blue, and residues 621 to 723 have been removed to allow an unobstructed view of the cork domain, which is shown in yellow. The LPS and ferrichrome-iron molecules are represented as balland-stick models, with the iron atom indicated as a large red sphere. Small red spheres are oxygen atoms, small white spheres are carbon atoms in the LPS molecule, small blue spheres are nitrogen atoms, small pink spheres are phosphorus atoms, and small black spheres are carbon atoms in the ferrichrome-iron molecule. FhuA is oriented as it would be found in the outer membrane; surface-located loops face the external environment (top), and periplasmic turns face the periplasm (bottom). Apices A $\left(\mathrm{Arg}^{81}\right), \quad B \quad\left(G \ln ^{100}\right)$, and C

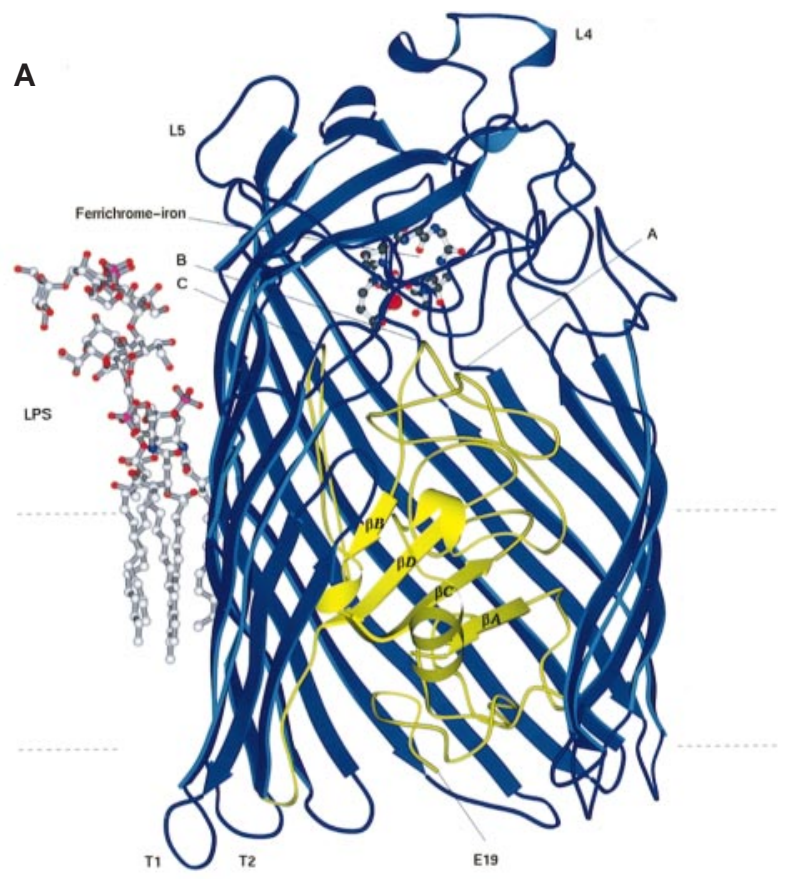

B

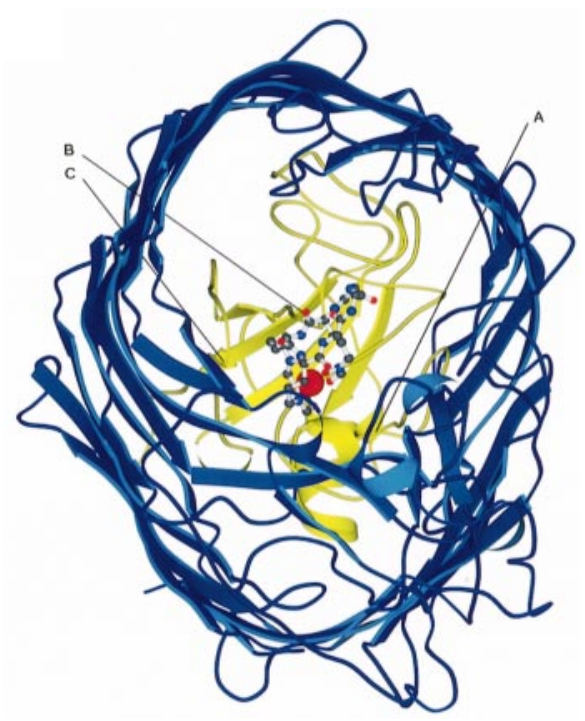

$\left(\mathrm{Tyr}^{116}\right) ; \beta A$ through $\beta D$ of the cork domain; surface-located loops [L4 (residues 318 to 339 ) and L5 (residues 402 to 428)]; periplasmic turns [T1 (residues 184 to 189) and T2 (residues 222 to 226)]; and $\mathrm{Glu}^{19}$ are labeled. The positions of the upper and lower aromatic girdles are indicated with dashed lines. The spaces above and below the cork domain are the external and periplasmic pockets, respectively. (B) FhuA as viewed from the external

environment along the barrel axis. The barrel is colored blue, and the cork domain is shown in yellow. The ferrichrome-iron molecule is represented as a ball-and-stick model, with the iron atom indicated as a large red sphere. In Fig. $1 B$ and in Fig. 5, A and B, small red spheres are oxygen atoms, small blue spheres are nitrogen atoms, and small black spheres are carbon atoms. Apices A, B, and C are labeled $(34,38)$. 
lation. The four-stranded $\beta$ sheet and the loops of the cork domain that are situated below apex $\mathrm{C}$ and the periplasmic pocket remain stationary (Fig. 4).

As a dramatic exception to the otherwise overall conservation of the secondary structure of FhuA upon ferrichrome-iron binding, a helix [termed the switch helix (residues 24 to 29)] that is located in the periplasmic pocket in the ligand-free conformation is completely unwound in the FhuA-ferrichrome-iron complex (Figs. 4 and 5B). The switch helix contains a number of inherently hydrophobic residues, and in the ligand-free conformation, it fits into a complementary hydrophobic pocket that is formed by select residues from $\mathrm{T} 8, \mathrm{~T} 9$, and $\beta \mathrm{A}$. Upon ferrichrome-iron binding, the upward translation of selected loops of the cork domain disrupts the interaction of this pocket with the hydrophobic face of the switch helix, thus promoting its destabilization. The stabilization of helices in short peptides due to interactions with hydrophobic side chains has been observed and theoretically discussed (18). All residues from $\mathrm{Arg}^{31}$ to the $\mathrm{NH}_{2}$-terminus $\left(\mathrm{Glu}^{19}\right)$ assume an extended conformation, bending $\sim 180^{\circ}$ in the opposite direction of the former helix axis. Glu $^{19}$ is placed near $\operatorname{Arg}^{128}$ from $\beta D$, in the center of the periplasmic pocket, 17.3 $\AA$ away from its former $\alpha$-carbon position (Figs. 4 and 5B) (19). All residues from Glu ${ }^{19}$ to the $\mathrm{NH}_{2}$ terminus, including the TonB box (residues 7 to 11), are disordered in both the FhuA and the
FhuA-ferrichrome-iron structures. As a result of this helix-coil transition, $\operatorname{Trp}^{22}$ occludes the periplasmic end of the putative channel-forming region, and the location of the TonB box in the ligand-bound conformation is changed (Fig. 5B) (20).

These observed allosteric transitions are in agreement with previous antibody recognition studies. All monoclonal antibodies that were bound to sequences between residues 21 to 59 discriminated between ligand-free and ligand-loaded FhuA (21). The incubation of purified FhuA with ferrichrome-iron, colicin $\mathrm{M}$, and (to a lesser extent) $\phi 80$ increased the relative amount of the FhuA-TonB complex that was cross-linked as compared to the amount of the FhuA-TonB complex that was cross-linked in the absence of ligand (22). The ability of these TonB-dependent FhuAspecific ligands to promote the physical interaction between FhuA and TonB suggests that allosteric transitions observed in the cork domain of the FhuA-ferrichrome-iron complex may be similar (notably, the unwinding of the switch helix). The unwinding of the switch helix is a clear periplasmically disposed conformational change, which signals the ligand-loaded status of the receptor and therefore the need for TonB-dependent energy transduction. Considering that siderophore receptors must compete for a limited amount of TonB (23), efficient signal transduction across the cell envelope to indicate the occu-

Table 1. Crystallographic data. FhuA and SeMet-FhuA were purified by immobilized ligand affinity chromatography with $0.05 \%$ dimethyldodecylamine- $N$-oxide and exchanged into $0.8 \%$ dimethyldecylamine- $N$-oxide (35). For SeMet-FhuA, $2.5 \mathrm{mM}$ of reduced glutathione was added to all buffers. Crystals of FhuA and SeMet-FhuA were grown with the hanging drop vapor diffusion technique by mixing $5 \mu \mathrm{l}$ of protein at $6.5 \mathrm{mg} / \mathrm{ml}$ with an equal volume of reservoir solution $[0.1 \mathrm{M}$ sodium cacodylate $(\mathrm{pH} 6.4), 11 \%$ polyethylene glycol (PEG), 2000 monomethyl ether, 20\% glycerol, 3\% PEG 200, 0.8\% dimethyldecylamine- $\mathrm{N}$-oxide, and $1 \%$ cis-inositol] (36). FhuA and SeMet-FhuA in complex with ferrichrome-iron ( $\mathrm{Fc}$ ) were crystallized under similar conditions. All crystals grew within 7 days to a final size of 350 by 350 by $350 \mu \mathrm{m}^{3}$ at $18^{\circ} \mathrm{C}$. They belonged to the primitive hexagonal space group $P 6_{1}(a=b=171.4 \AA ; c=$ $85.7 \AA$ ), with one molecule per asymmetric unit, a Matthews coefficient of $4.82 \AA^{3} /$ dalton, and a solvent content of $74.3 \%$. Native data were collected at $100 \mathrm{~K}$ from flash-frozen crystals of FhuA and SeMet-FhuA complexed with Fc to resolutions of 2.5 and $2.7 \AA$, respectively. MAD data were collected from a SeMet-FhuA-Fc complex crystal to a resolution of $3.05 \AA$, allowing the structure to be solved. For details of structure solution and refinement, see (37). Parentheses denote the highest shell. Phasing power is the mean value of heavy atom structure factor amplitude divided by lack of closure. $R_{\text {sym }}=$ $\Sigma_{h k l} \Sigma_{i}\left|I_{h k l}-\left\langle I_{h k l}\right\rangle\right| / \Sigma_{h k l} \Sigma_{i} I_{h k l}$, where $\left\langle I_{h k l}\right\rangle$ is the average of symmetry-related $I_{h k l}$.

\begin{tabular}{|c|c|c|c|c|c|}
\hline \multirow[b]{2}{*}{ Diffraction data } & \multirow[b]{2}{*}{ FhuA } & \multirow{2}{*}{$\begin{array}{l}\text { SeMet- } \\
\text { FhuA- } \\
\text { complex Fc }\end{array}$} & \multicolumn{3}{|c|}{ MAD } \\
\hline & & & Remote & $\begin{array}{l}\text { Point of } \\
\text { inflection }\end{array}$ & Peak \\
\hline Wavelength $(\AA)$ & 1.051 & 1.051 & 0.8876 & 0.9782 & 0.9779 \\
\hline Resolution $(\AA)$ & $\begin{array}{c}2.45 \\
(2.45-2.50)\end{array}$ & $\begin{array}{c}2.70 \\
(2.70-2.80)\end{array}$ & $\begin{array}{c}3.05 \\
(3.05-3.10)\end{array}$ & $\begin{array}{c}3.05 \\
(3.05-3.10)\end{array}$ & $\begin{array}{c}3.05 \\
(3.05-3.10)\end{array}$ \\
\hline Unique reflections & $\begin{array}{l}53,749 \\
(2,931)\end{array}$ & $\begin{array}{l}39,633 \\
(4,038)\end{array}$ & $\begin{array}{l}26,521 \\
(1,268)\end{array}$ & $\begin{array}{l}27,448 \\
(1,282)\end{array}$ & $\begin{array}{l}27,233 \\
(1,268)\end{array}$ \\
\hline Completeness (\%) & $99.3(92.2)$ & $98.9(98.0)$ & $96.6(99.3)$ & $99.5(98.9)$ & $98.8(97.8)$ \\
\hline Redundancy & $6.4(3.1)$ & $5.9(3.7)$ & $8.4(6.8)$ & $6.4(2.8)$ & $5.2(2.3)$ \\
\hline$R_{\text {sym }}(\%)$ & $7.8(33.4)$ & $7.9(40.6)$ & $14.6(67.8)$ & $11.0(46.8)$ & $11.4(42.0)$ \\
\hline \multicolumn{6}{|c|}{ Phasing power (acentric) } \\
\hline Dispersive & - & - & 2.27 & 0 & 1.85 \\
\hline Anomalous & - & - & 1.43 & 1.45 & 1.75 \\
\hline Figure of merit & - & - & - & 0.45 & - \\
\hline
\end{tabular}

pancy of the receptor is essential for the physiology of the bacterial cell (24).

A mechanism of ferrichrome-iron transport. A model for the transport of siderophores by TonB-dependent receptors is necessarily subject to constraints imposed by structural data, studies of ligand binding, phenotypes of genetic mutants, and residue conservation among different FhuAs. We propose the following basic model. After the initial physical interaction between ferrichrome-iron and the surface-located loops of FhuA, the ligand is partitioned from the external medium into the external pocket by its affinity for aromatic residues. It is then bound with high affinity by an induced fit mechanism, resulting in an allosteric transition. Subsequent transport of ferrichromeiron to the periplasm is dependent on the disruption of the binding site. We propose that the formation of the FhuA-TonB complex and the subsequent energy transduction induce a further allosteric transition to reduce the stability of the ferrichrome-iron binding site. A disruption of the induced fit binding mechanism may be effected by a small shift of apices A, B, and C toward the periplasm as a consequence of energy being transduced by TonB.

When viewed along the barrel axis, the external pocket is connected to the periplasmic pocket in one segment of the barrel cross section by a narrow water-filled channel. We designated this segment as the putative channel-forming region (Fig. 5A). Located directly below apex B is a short coil containing the strongly conserved residues Leu ${ }^{106}, \mathrm{Asn}^{107}$, and Gly ${ }^{108}$ (12). Subtle conformational changes of this and other loops of the cork domain between apex B and the periplasmic pocket of FhuA would suffice to allow the permeation of ferrichrome-iron through the putative channel-forming region (Figs. 4 and $5 \mathrm{~A})$. We therefore propose that, after the formation of the FhuA-TonB complex, a channel opens in this region by the rearrangement of loops of the cork domain.

Among TonB-dependent receptors, there are few regions of strict sequence conservation. However, sequence alignments of FhuA proteins identify a series of strongly conserved residues (25) that are positioned on the inner barrel wall of the putative channel-forming region. These residues coat an extended inner barrel surface from the ferrichrome-iron binding site to the periplasmic pocket of FhuA (Fig. $5 \mathrm{~A})$. The arrangement of these residues may function as a series of low-affinity binding sites for the surface diffusion of ferrichrome-iron through FhuA. The weak adsorption of ferrichrome-iron to the inner barrel wall could mediate both the rapid diffusion (26) of the ligand inside the putative channel-forming region and, by its binding, confinement to this region.

This proposal is supported by previous studies involving channel-forming deletion 
mutants of FhuA. The excision of some surface-located linear sequences from TonB-dependent siderophore receptors resulted in the formation of nonspecific open diffusion channels. Specifically, the deletion of residues 322 to 355 (residues from $\beta 7$, L4, and $\beta 8$ ) and 335 to 355 (residues from L4 and $\beta 8$ ) from FhuA abolished TonB-dependent ferrichrome-iron transport while permitting the nonspecific diffusion of ferrichrome-iron, sodium dodecyl sulfate, and maltodextrins across the outer membrane $(5,27,28)$. Small

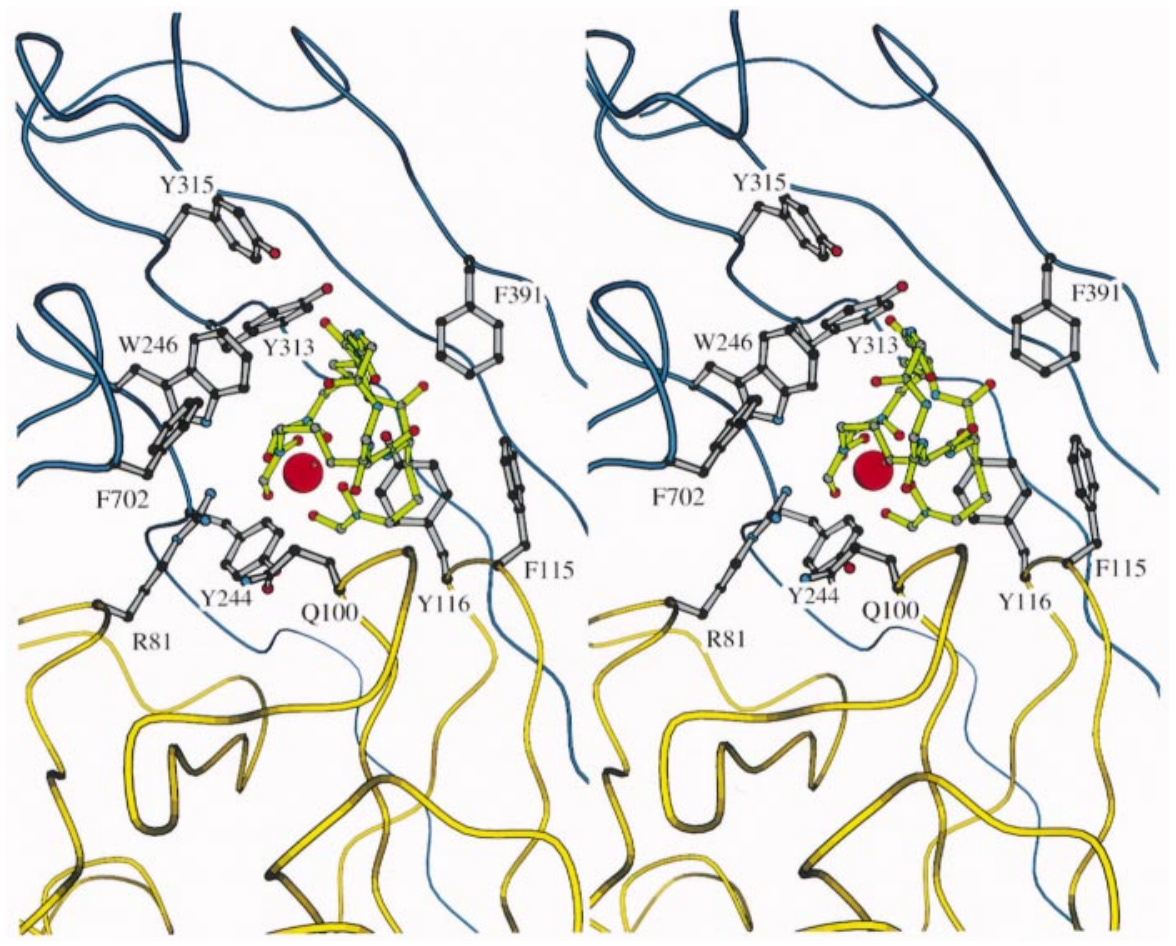

Fig. 2. Stereoview of the ferrichrome-iron binding site in ribbon representation. The ferrichromeiron molecule is represented as a green ball-and-stick model, with the iron atom indicated as a red sphere. Small red spheres are oxygen atoms, small blue spheres are nitrogen atoms, and small black spheres are carbon atoms of select side-chain residues of FhuA, and small gray spheres are carbon atoms of the ferrichrome-iron molecule. The cork domain is shown in yellow; barrel strands and loops are shown in blue. Side-chain residues (Arg ${ }^{81}$ from apex A, Gln ${ }^{100}$ from apex B, Phe ${ }^{115}$ and $\mathrm{Tyr}^{116}$ from apex C, Tyr ${ }^{244}$ and $\operatorname{Trp}^{246}$ from L3, $\operatorname{Tyr}^{313}$ and $\mathrm{Tyr}^{315}$ from $\beta 7$, Phe ${ }^{391}$ from $\beta 9$, and Phe $^{702}$ from $\beta 21$ ) within $4.5 \AA$ of ferrichrome-iron atoms are labeled (34) and shown in gray.

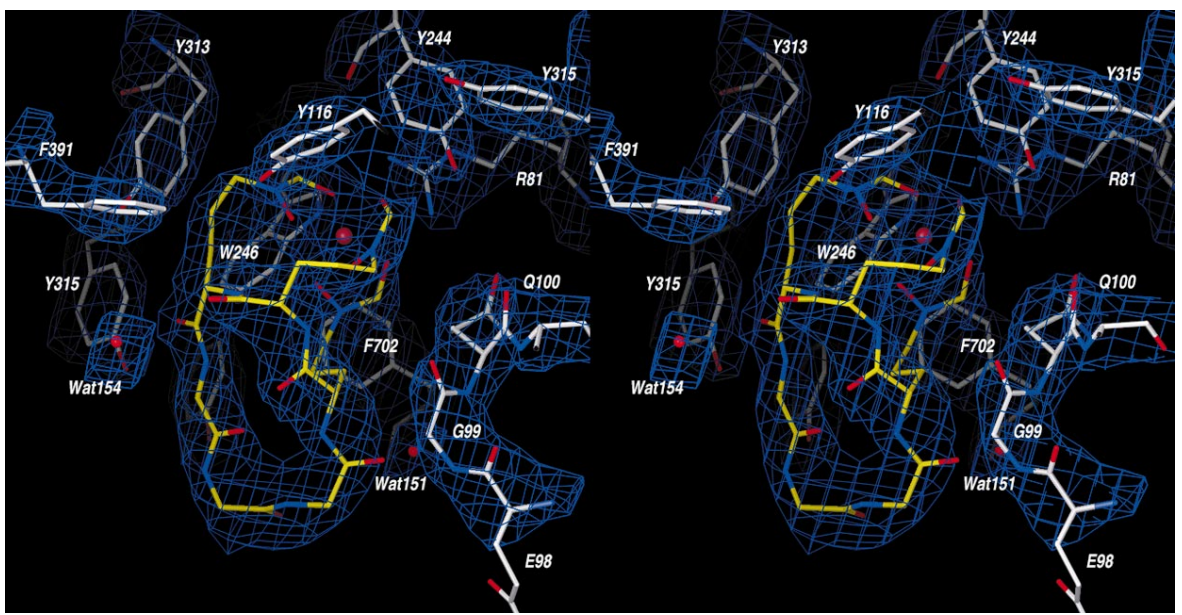

Fig. 3. Representative section of the electron density map. Stereoview of the final $3 F_{\text {obs }}-2 F_{\text {calc }}$ electron density map (blue) at a resolution of $2.7 \AA$ is contoured at $1.5 \sigma$, showing the ferrichrome-iron binding site, including water molecules. The ferrichrome-iron molecule is shown in yellow, and the iron atom is indicated as a large red sphere. Select side-chain residues (Glu ${ }^{98}$, $\mathrm{Gly}^{99}$, and Gln ${ }^{100}$ from apex B; Tyr ${ }^{116}$ from apex C; $\operatorname{Tyr}^{244}$ and $\operatorname{Trp}^{246}$ from L3; Phe ${ }^{313}$ from $\beta 7$; $\mathrm{Phe}^{391}$ from $\beta 9$; and Phe ${ }^{702}$ from $\beta 21$ ) and the two water molecules (Wat151 and Wat154) found in the binding site are labeled (34) and colored white and red, respectively. fluctuations in the conductance patterns of channel-forming FhuAs were observed; they were different from those of porins and possibly resulted from changes in the cross-sectional diameter of the channel. The binding of bacteriophage T5 to FhuA induced similar channels (7). Considering the structure, the removal of residues 340 to 355 from $\beta 8$ would disrupt critical connections between the barrel wall and apex $\mathrm{C}$ of the cork domain, resulting in a higher degree of conformational flexibility of the coil segments around apices $\mathrm{B}$ and $\mathrm{C}$ and of more remote regions of the cork domain. The result may be the transient opening and closing of an aqueous channel (Fig. 5A). The location and structure of the channel may be similar to that formed in vivo by the FhuA-TonB complex. This suggestion is supported by planar lipid bilayer experiments involving the FhuA-bacteriophage T5 complex. The addition of ferrichrome-iron to either chamber of the bilayer apparatus resulted in a reduction in channel conductance; ferrichrome-iron may have bound to a distorted binding site (7).

We postulate that ferrichrome-iron is liberated from its high-affinity binding site and diffuses to the periplasm through a channel similar in structure and size to that induced by the binding of bacteriophage T5. This surface diffusion model resembles the model that was postulated for the permeation of

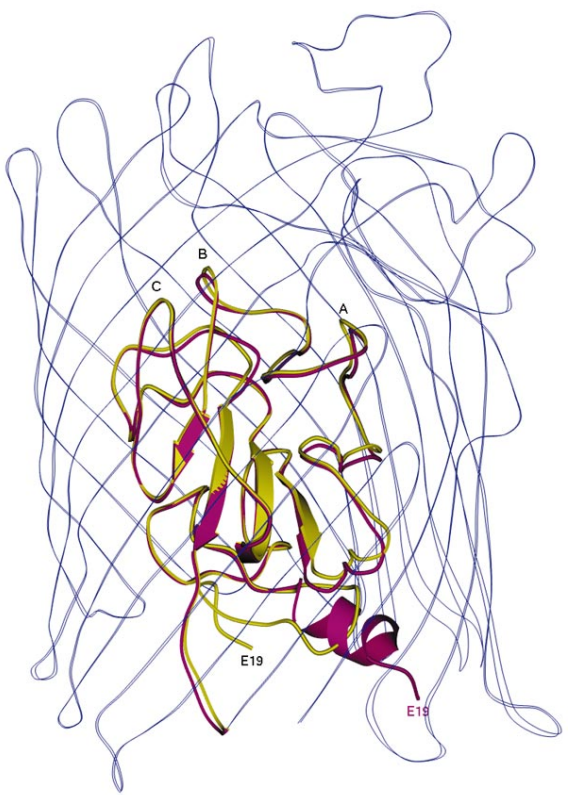

Fig. 4. Conformational changes induced upon ferrichrome-iron binding. Superposition of the $\alpha$-carbon coordinates of FhuA and its complex with ferrichrome-iron illustrating the ligandinduced conformational changes observed in the cork domain. The cork domains of FhuA and its complex with ferrichrome-iron are shown in purple and yellow, respectively. The barrel strands (shown in blue) are represented as thin lines for clarity of the cork domain. Apices A, B, and $\mathrm{C}$ and $\mathrm{Glu}^{19}$ are labeled (34). 
sugars through the glycoporins (29). When ferrichrome-iron reaches the periplasmic pocket of FhuA, it is bound by the highaffinity periplasmic binding protein FhuD $\left(K_{\mathrm{D}}, 0.1 \mu \mathrm{M}\right)(1,30)$, thereby ensuring unidirectional transport across the cell envelope (31). We further postulate that other TonBdependent siderophore receptors undergo similar ligand-induced allosteric transitions, transport their cognate siderophore through channels by surface diffusion, and therefore utilize a common siderophore-mediated iron transport mechanism.

This proposed mechanism suggests an explanation for the evolution of high-affinity receptors for different siderophore-iron complexes by Gram-negative bacteria. Only the external aromatic pocket and the high-affinity binding site must be tailored to different ligands. Ligand-induced allosteric transitions and transport are common mechanistic features and are essentially receptor independent. The inherent flexibility of this design is advantageous in adapting siderophore receptors such as FhuA for the fungal siderophore ferrichrome (which is an obvious advantage, given variations in iron supply for bacteria). Moreover, the correlation between bacterial virulence in vivo and the expression of highaffinity TonB-dependent iron acquisition systems, including receptors for transferrin, lactoferrin, and heme and ferric siderophores $(1$, 32 ), indicates adaptations that allow bacteria to survive in the interstitial spaces and the bloodstream of host organisms. The high affinity and specificity of TonB-dependent sid-
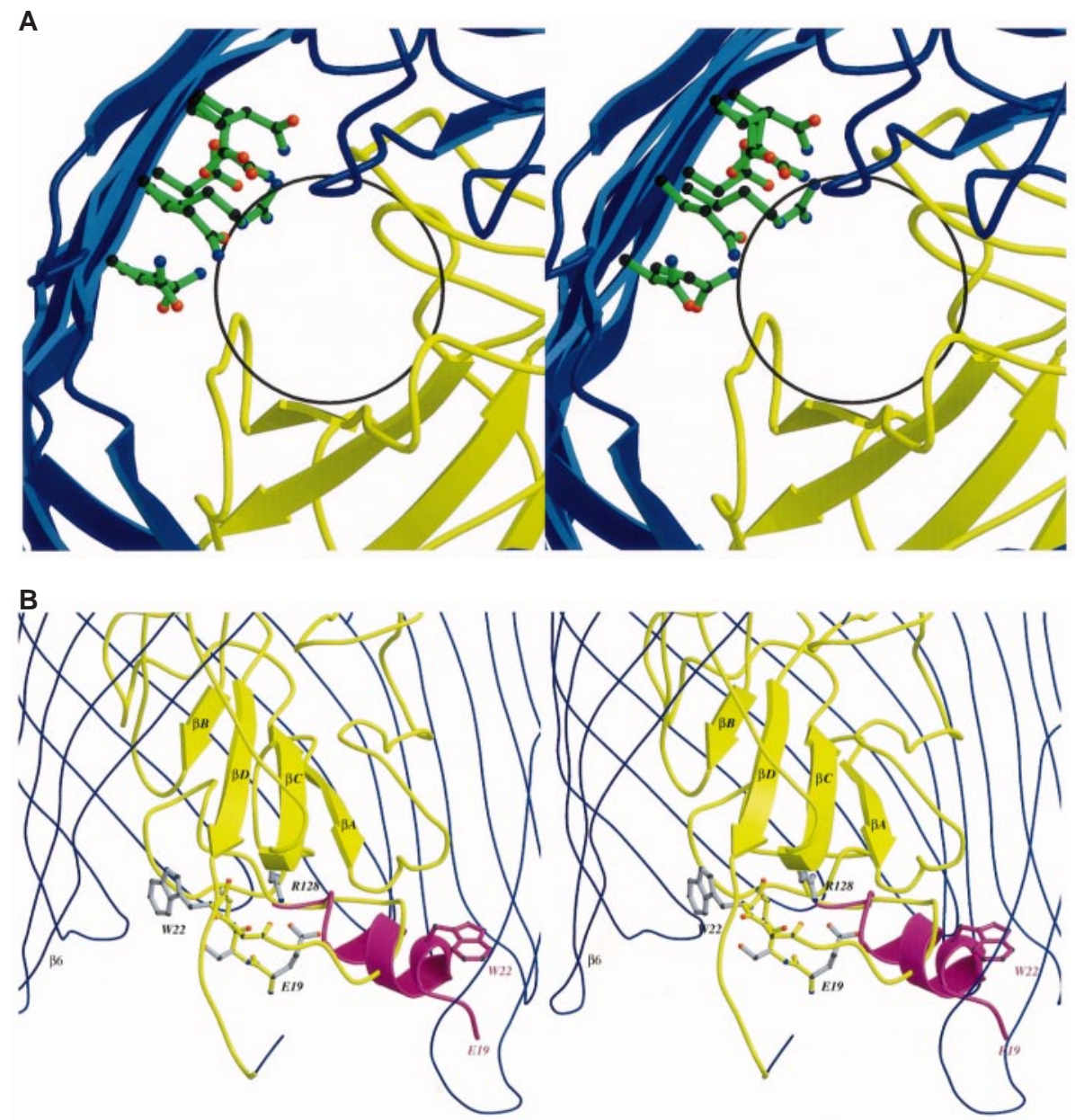

Fig. 5. The putative channel-forming region with bound ferrichrome-iron complexes. (A) Stereoview of the putative channel-forming region in ribbon representation as viewed from the external environment along the barrel axis. This is an enlargement of the upper left portion of Fig. 1B. The barrel is colored blue, and the cork domain is shown in yellow. The putative channel-forming region is indicated by a circle (diameter, $10 \AA$ ). Strictly conserved side-chain residues ( $\operatorname{Arg}^{297}$ and $\mathrm{Asn}^{299}$ from $\beta 7$; Asp ${ }^{358}$ $G \ln ^{360}$, and $A s p^{379}$ from $\beta 9$; and $A s n^{436}, G \ln ^{438}$, and $G \ln ^{440}$ from $\beta 10$ ) that are thought to be involved in the surface diffusion of ferrichrome-iron complexes through the putative channel-forming region are shown in green. (B) Stereoview illustrating the unwinding of the switch helix as a result of an allosteric transition that was induced upon ferrichrome-iron binding. The barrel strands (shown in blue) are represented as thin lines for clarity. Cork domain strands $\beta A$ through $\beta D, G^{19}{ }^{19} \operatorname{Trp}^{22}$, and $\operatorname{Arg}^{128}$ are labeled (34). The switch helix in the ligand-free conformations and the coil in the ligand-loaded conformations are shown in purple and yellow, respectively.

erophore receptors make them ideal targets for the design of novel antibacterial agents such as siderophore-antibiotic conjugates (33). The principle relies on the specific recognition of the outer membrane receptor by the siderophore moiety, thereby ensuring transport of the conjugate through the receptor and into the periplasm of the bacterial cell.

\section{References and Notes}

1. V. Braun, K. Hantke, W. Köster, in Metal lons in Biological Systems, A. Sigel and H. Sigel, Eds. (Marcel Dekker, New York, 1998), pp. 67-145.

2. W. Welte et al., in Proceedings of the NATO Advanced Workshop: New Methods for the Study of Molecular Aggregates, K. Standing and W. Ens, Eds. (Kluwer Academic, Boston, 1998), pp. 239-276; W. Welte, U. Nestel, T. Wacker, K. Diederichs, Kidney Int. 48, 930 (1995).

3. K. Postle, Mol. Microbiol. 4, 2019 (1993); T. E. Letain and K. Postle, ibid. 24, 271 (1997).

4. J. W. Coulton et al., J. Bacteriol. 165, 181 (1986).

5. H. Killmann, R. Benz, V. Braun, EMBO. J. 12, 3007 (1993).

6. J. M. Rutz et al., Science 258, 471 (1992).

7. M. Bonhivers et al., EMBO. J. 15, 1850 (1996).

8. L. Holm and C. Sander, J. Mol. Biol. 233, 123 (1993); K. Diederichs, Proteins Struct. Funct. Genet. 23, 187 (1995).

9. C. R. H. Raetz, in Escherichia coli and Salmonella Cellular and Molecular Biology, F. C. Neidhardt, Ed. (American Society for Microbiology Press, Washington, DC, 1996), pp. 1035-1071.

10. Crystallization of FhuA is dependent on the presence of stoichiometric amounts of LPS. If LPS is completely removed from FhuA protein preparations or if an excess of LPS is present in such preparations, the growth of FhuA crystals is inhibited. We propose that LPS remained bound to FhuA throughout the process of purification and crystallization and that it did not adsorb to FhuA during isolation. Because it is known that LPS is localized to the outer leaflet of the outer membrane, the location of bound LPS marks its position relative to the upper aromatic girdle of FhuA and to the outer membrane.

11. Strongly conserved residues found in the ferrichrome-iron binding site are $\mathrm{Arg}^{81}$ from apex A, Gly ${ }^{99}$ and $\mathrm{Gln}^{100}$ from apex B, Phe ${ }^{115}$ and $\mathrm{Tyr}^{116}$ from apex C, Tyr ${ }^{244}$ and $\operatorname{Trp}^{246}$ from L3, Tyr ${ }^{313}$ and $\mathrm{Tyr}^{315}$ from $\beta 7$, Phe ${ }^{391}$ from $\beta 9$, and $\mathrm{Phe}^{702}$ from $\beta 21$.

12. H. Killmann et al., J. Bacteriol. 180, 3845 (1998)

13. The binding site possesses a higher affinity for ferrichrome-iron than for apoferrichrome [P. Boulanger et al., Biochemistry 35, 14216 (1996)]. In the ligandloaded structure, $\mathrm{Tyr}^{244}$ comes in close contact with the iron atom of the ferrichrome-iron molecule. This observation may explain the decreased affinity for apoferrichrome.

14. A single amino acid deletion, $\Delta \operatorname{Asp}^{348}[\mathrm{H}$. Killmann and V. Braun, J. Bacteriol. 174, 3479 (1992)], inhibited ferrichrome-iron binding and the transport activity of FhuA.

15. Residues lining the external aromatic pocket are $\mathrm{Tyr}^{325}$ from L4, Tyr ${ }^{342}$ from $\beta 8, \mathrm{Tyr}^{393}$ from $\beta 9$, $\mathrm{Phe}^{566}$ and $\mathrm{Phe}^{567}$ from L8, Tyr ${ }^{610}$ from L9, Tyr ${ }^{705}$ from L11, and Phe ${ }^{708}$ from $\beta 22$.

16. J. B. Neilands, J. Am. Chem. Soc. 74, 4846 (1952).

17. D. A. Dougherty, Science 271, 163 (1996).

18. D. Butcher, M. D. Bruch, G. R. Moie, Pept. Biopolym. 35, 109 (1995); A.-S. Yang and B. Honig, J. Mol. Biol. 252, 351 (1995).

19. The insertion of a dipeptide into the fhuA gene after residue $\operatorname{Arg}^{128}$ results in a complete loss of all FhuA function [G. Carmel et al., J. Bacteriol. 172, 1861 (1990)].

20. The tryptophan emission spectra were measured for FhuA and the FhuA-ferrichrome-iron complex [K. Locher and J. Rosenbusch, Eur. J. Biochem. 247, 779 (1997)]. The tryptophan fluorescence was shown to decrease after the addition of the ferrichrome-iron, which suggests a change in accessibility. 
21. G. S. Moeck et al., Mol. Microbiol. 22, 459 (1996).

22. G. S. Moeck, J. W. Coulton, K. Postle, J. Biol. Chem. 272, 28391 (1997).

23. P. E. Klebba et al., J. Bioenerg. Biomembr. 25, 603 (1993); R. J. Kadner and K. J. Heller, J. Bacteriol. 177, 4829 (1995).

24. G. S. Moeck and J. W. Coulton, Mol. Microbiol. 28, 675 (1998).

25. Strictly conserved side-chain residues that are thought to be involved in the surface diffusion of ferrichrome-iron through the putative channel-forming region may be $\operatorname{Arg}^{297}$ and $A_{s n^{299}}$ from $\beta 7 ; A_{s p}{ }^{358}$, $\mathrm{Gln}^{360}$, and $\mathrm{Asp}^{379}$ from $\beta 9$; and $\mathrm{Asn}^{436}, \mathrm{Gln}^{438}$, and $\mathrm{Gln} 440$ from $\beta 10$.

26. G. Adam and M. Delbrück, in Structural Chemistry and Molecular Biology, A. Rich and N. Davidson, Eds. (Freeman, San Francisco, 1968), pp. 198-215.

27. H. Killmann, R. Benz, V. Braun, J. Bacteriol. 178, 6913 (1996).

28. Strand $\beta 7$ is composed of residues 294 to $317, L 4$ is composed of residues 318 to 339 , and strand $\beta 8$ is composed of residues 340 to 366 .

29. T. Schirmer et al., Science 267, 512 (1995); D. Forst et al., Nature Struct. Biol. 5, 37 (1998).

30. A. Mademidis et al., Mol. Microbiol. 26, 1109 (1997).

31. A more complicated mechanism could avoid the possible loss of the ferrichrome-iron into the external medium after the formation of the FhuA-TonB complex by the steric blockage of the surface-located pocket. However, the TonB-dependent binding and uptake of the FhuA-specific toxin colicin $M$ through FhuA would require that the putative channel-forming region remain open from the external environment to the periplasm for an extended period of time and therefore would contradict such a mechanism [C. J. Lazdunski et al., J. Bacteriol. 180, 4993 (1998); R. M. Stroud et al., Curr. Opin. Struct. Biol. 8, 525 (1998)]

32. M. L. Guerinot, Annu. Rev. Microbiol. 48, 743 (1994).

33. A. Ghosh et al., Chem. Biol. 3, 1011 (1996).

34. Single-letter abbreviations for the amino acid residues are as follows: A, Ala; C, Cys; D, Asp; E, Glu; F, Phe; G, Gly; H, His; I, Ile; K, Lys; L, Leu; M, Met; N, Asn; P, Pro; Q, Gln; R, Arg; S, Ser; T, Thr; V, Val; W, Trp; and Y, Tyr.

35. Because FhuA is difficult to purify to homogeneity in large amounts, one prerequisite for crystallization and subsequent structural analysis by $\mathrm{x}$-ray crystallography was the development of an overexpression system and efficient protocols for the rapid purification of FhuA [A. D. Ferguson et al., Protein Sci. 7, 1636 (1998)]. A recombinant FhuA was constructed by splicing a hexahistidine tag and three additional residues (SHHHHHHGS) (34) at a previously identified surface location (amino acid 405) [G. S. Moeck et al., J. Bacteriol. 176, 4250 (1994)]. Functional assays confirmed (21) that the protein FhuA405. $\mathrm{H}_{6}$ (native FhuA) is fully active as a bacteriophage receptor and is also active for TonBdependent ferrichrome-iron transport at levels comparable to those of the wild type. To generate selenomethionyl-labeled FhuA (SeMet-FhuA), plasmid pHX405 was transformed into the met auxotrophic $E$. coli strain DL41 and grown as recommended [S. Doublié, Methods Enzymol. 276, 523 (1997)].

36. S. J. Angyal, L. Odier, M. E. Tate, Carbohydr. Res. 266, 143 (1993).

37. Phase information was derived from multiple anomalous dispersion (MAD) [W. A. Hendrickson, Science 254, 51 (1991)] data that were measured at three wavelengths that corresponded to the point of inflection, the peak of the selenium absorption profile, and a remote point. All data were reduced and processed with the XDS software package [W. Kabsch, J. Appl. Crystallogr. 21, 916 (1988)]. The 10 selenium sites were located with SOLVE [T. C. Terwilliger and J. Berendzen, Acta Crystallogr. D53, 571 (1997)], and MAD phases were obtained with SOLVE and SHARP [E. de La Fortelle and G. Bricogone, Methods Enzymol. 276, 472 (1997)]. Initial phases, which were calculated to a resolution of $3.05 \AA$, were improved by solvent flattening with the program DM [K. Cowtan, Acta Crystallogr. D50, 760 (1994)]. The resulting electron density maps were of sufficient quality to build a model with the program O [T. A. Jones et al. Acta Crystallogr. A47, 110 (1991)] and unambiguously place a ferrichrome-iron molecule and a LPS molecule. The model was refined with the programs X-PLOR [A. T. Brünger, X-PLOR 3.1 (Yale University, New Haven, CT, 1992)] and CNS [A. T. Brünger et al., Acta Crystallogr. D54, 905 (1998)] and was used to solve the structure of FhuA to a resolution of $2.5 \AA$ by difference Fourier techniques. Standard protocols for simulated annealing and minimization as implemented in CNS were used for refinement. Individual restrained B-factor refinement was justified as judged by a substantial drop in $R_{\text {free }}$ [A. T. Brünger, Nature 355, 472 (1992)]. The current FhuA model contains residues 19 to 723,1 LPS, and 99 water molecules. The average $B$ factors for main-chain and side-chain atoms and the LPS molecule are 65,67 , and $78 \AA^{2}$, respectively. The FhuA model was used in the refinement of the FhuA-ferrichrome-iron complex to a resolution of $2.7 \AA$. The current model for the FhuAferrichrome-iron complex contains residues 19 to 723, 1 LPS, 1 ferrichrome-iron molecule, and 52 water molecules. The average B factors for mainchain and side-chain atoms, the LPS, and ferrichrome-iron molecules are $63,66,75$, and $49 \AA^{2}$ respectively. Refinement is not complete for both structures. For the FhuA model, the $R$ is $24.2 \%$ (48,359 reflections), and the $R_{\text {free }}$ is $28.3 \%$ (2309 reflections); for the FhuA-ferrichrome-iron complex, the $R$ is $23.2 \%$ (37,362 reflections), and the $R_{\text {fre }}$ is $28.1 \%$ (1532 reflections). All residues lie in allowed regions of the Ramachrandran plot, and all residues that are explicitly mentioned in the text reside in good electron density. Protein Data Bank accession codes are $2 \mathrm{fcp}$ and $1 \mathrm{fcp}$ for FhuA and the FhuAferrichrome-iron complex, respectively.

38. All figures were prepared with the programs MOLSCRIPT [P. Kraulis, J. Appl. Crystallogr. 24, 946 (1991)] and Raster-3D [E. A. Merrit and D. J. Bacon, Methods Enzymol. 277, 505 (1997)], except for Fig. 3, which was prepared with the program $O$.

39. We gratefully acknowledge A. Svensson at MAX-lab II and A. Thompson at the European Radiation Synchrotron Facility for their assistance and generous support during data collection; E. A. Meighen for providing $E$. coli strain DL41; P. A. Karplus for a critical reading of the manuscript; J. Wang for genetic constructs; $V$. Braun and $H$. Killmann for bacterial strains and discussions; A. Patel for his assistance with protein purification; J. Breed for crystallization trials and a critical reading of the manuscript; D. M. Allan and J. A. Kashul for editing, K. Hegetschweiler for providing cis-inositol; and B. Herrmann, A. Hirsch, C. Peinelt, $\mathrm{O}$. Seth, and J. Telioriclis, who made important contributions to the early phase of this project. This work was supported by the Deutsche Forschungsgemeinschaft (W.W.); by the Medical Research Council, Canada (grant MT-14133 to J.W.C.); and by NATO International Collaborative Research grant 960082 . A.D.F. is the recipient of a Deutscher Akademischer Austauschdienst Grant for Study and Research.

23 October 1998; accepted 13 November 1998 